\title{
An Error Analysis into the Use of Regular Targets and Target Detection in Image Analysis for Impact Engineering
}

\author{
A. Jumpasut ${ }^{1, a}$, N. Petrinic ${ }^{1, b}$, B. C. F. Elliott ${ }^{1, c}$, C. R. Siviour ${ }^{1, d}$ and M. R. \\ Arthington ${ }^{1, e}$ \\ ${ }^{1}$ University of Oxford, Department of Engineering Science, Parks Road, Oxford, OX1 3PJ, UK \\ aarin.jumpasut@eng.ox.ac.uk, bnik.petrinic@eng.ox.ac.uk, cben.elliott@eng.ox.ac.uk, \\ dclive.siviour@eng.ox.ac.uk, ${ }^{\mathrm{e}}$ matthew.arthington@eng.ox.ac.uk
}

Keywords: Impact engineering, high-speed photography, image analysis, photogrammetry, bulge experiments

\begin{abstract}
This study concentrates on the use of corners targets for photogrammetry in impact engineering. An example of high speed experimentation is presented and the associated difficulties are discussed. The relevant corner detection methods that have been implemented and developed are investigated and their accuracy assessed. This study focuses solely upon the effect of blurring on the accuracy of the detection methods; it is part of a much wider investigation into the use and accuracy of different targets and target detection methods for photogrammetry in impact engineering. A set of tests has been performed and the errors between the true position of the corner and the detected position are compared.
\end{abstract}

\section{Introduction}

Advances in numerical modelling of a material's response to loading, particularly in the case of heterogeneous materials such as composites, geological and biological materials, as well as the use of data hungry inverse modelling strategies have led to an increasing requirement for full field measurements of specimen behaviour during experimental characterisation. Furthermore, in extreme conditions such as loading at high rates of strain or at extreme temperatures [1], it is not physically possible to use contacting sensors such as strain gauges to provide information about specimen behaviour. Even when this may be possible, contacting sensors can only provide limited information. For this reason, there is great interest in the use of photogrammetry to calculate specimen deformation fields from images taken using microscopy, photography or even X-ray imaging.

This paper will first describe various methods available that can help locate the position of a corner in an image to a high degree of accuracy. It will then describe an example of an analysis of a high speed experiment and the difficulties that arose. The work analysing the results of this experiment inspired a wider investigation and error analysis into targets and target detection methods, which included stripes, dots and corners as targets. This error analysis studied the effect of blurring, different resolutions, shot noise and detector noise on the ability of various methods to locate the targets. This paper will describe some results from this study that was performed within a wider programme of work exploring the use of photogrammetry in impact engineering. For the purposes of this paper, 'corner' refers to a specific type of corner and is defined as the intersection of two lines at right angles to each other, with the bottom left and top right quadrants shaded pure black and the others pure white, as might be applied to a specimen prior to an experiment.

\section{Corner detection methods}

Five methods of finding the position of a corner in an image are included in this study. Four of them are based on cross correlating the image with a template and the last method is the Harris corner detector. 
Obtaining a discrete cross correlation surface. Cross correlation can be used to detect and identify the position of most types of targets. Cross correlation is a means of measuring the similarity between two images (or general matrices). This is especially useful in digital speckle analysis [2] but in this study is applied to non-random targets, namely corners. The discrete correlation of two matrices, $h_{s u b 1}$ and $h_{s u b 2}$, is defined as:

$$
c(k, l)=\sum_{i=0}^{m-1} \sum_{j=0}^{m-1}\left[h_{2}^{*}(i, j) h_{1}(k+i, l+j)\right] \text { for } k, l=0,1, \ldots, m-1
$$

where $h_{1}$ and $h_{2}$ are typically the matrix representations of the two images that are being compared, these are padded to size $m$ so that errors from the periodicity of the correlation function are avoided and ${ }^{*}$ denotes the complex conjugate. Calculating a correlation surface, $c$, in this manner is a slow process and can be speeded up significantly if it is performed in the Fourier domain.

$$
c(k, l)=\mathfrak{J}^{-1}\left(H_{2}^{*} H_{1}\right)
$$

where $H_{1}$ and $H_{2}$ are the Fourier transforms of $h_{1}$ and $h_{2}$. The whole function now consists of two Fourier transforms, a multiplication and an inverse Fourier transform, which greatly improves performance. After cross correlating two images (an image and a template, or an image and a section of the previous image), you obtain a correlation surface. The position of the area of the image most similar to the template is given by the peak of this correlation surface.

Finding the corner to a subpixel resolution. Finding the peak of a correlation surface will give the location of the target to the nearest pixel, since the correlation surface is discrete. To find the position to subpixel resolution various other methods may be applied and [2] suggests several methods of finding the position to subpixel resolution, including: expanding the correlation surface using a Fourier series and fitting a Gaussian to the correlation peak. These two methods were used in the current research along with a third method developed in house.

The explanation and derivation of how a Fourier series is used to expand the discrete correlation surface, Eq. (1), into a continuous correlation surface that can be used to find the correlation peak to sub pixel resolution, is given in [2]. The peak of this continuous correlation surface will provide the location of the corner to subpixel resolution.

The second method, mentioned in [2], and implemented in this study was to fit a Gaussian function to the discrete correlation peak. The mean of this Gaussian function will then provide the subpixel position of the template.

The final way of finding the peak of a correlation surface to a subpixel accuracy that was implemented for this study, is to use the magnitude of the pixels on the correlation surface that make up the correlation peak and to find the centroid of the correlation peak using the squared centroiding equation given in Eq. 3.

$$
\text { Squared centroiding of the correlation surface: }\left[\begin{array}{c}
k_{0} \\
l_{0}
\end{array}\right]=\frac{\sum_{i=1}^{n} c^{2}\left(k_{i}, l_{i}\right)\left[\begin{array}{c}
k_{i} \\
l_{i}
\end{array}\right]}{\sum_{i=1}^{n} c^{2}\left(k_{i}, l_{i}\right)}
$$

This error analysis will consider the use the following methods of locating a corner: the discrete cross correlation peak, continuous cross correlation peak (using a Fourier series to expand the discrete correlation surface), fitting a Gaussian function to the discrete correlation peak and the use of a squared centroiding algorithm on the peak of the discrete correlation surface. The Matlab based in house implementation of these target location methods were used in this study. All of these methods have already been used in practice, [3], except for the squared centroiding algorithm on the discrete correlation peak, which has not yet been encountered in the literature.

Harris corner detector. Feature detection is also an important task for computer vision and there exist sophisticated target detectors which are used regularly in this field. One of these is the Harris 
detector [4]. This attempts to detect corners by taking an image patch and considering the difference in intensity when this image patch is shifted in different directions. A patch of solid colour will have little or no difference when shifted in either the vertical or horizontal direction. A vertical edge will have little or no difference when shifted in the vertical direction but have a large difference when shifted in the horizontal direction and vice versa for a horizontal edge. A corner will have large differences when shifted in both directions. The Harris corner detector is used commonly in computer vision, often in conjunction with other constraints or algorithms [5]. For the purpose of this error analysis, we have used an implementation of the Harris corner detector from the 'Camera Calibration Toolbox for Matlab' [6] although many other implementations exist.

\section{An example of using the results of corner detection in a high speed experiment}

Biaxial tension (bulge) tests are important tests in material characterisation and as such are invaluable in accurate modelling. A thin plate specimen of Ti6Al4 was placed over a chamber of oil and the pressure of the oil was increased at a high rate until the specimen bulged and then burst. This was filmed by a high speed camera at 13,000 frames per second and two mirrors were used in order to provide two views of the bulge, which could be used later to reconstruct the geometry of the bulge. A grid of corners was used to mark the specimen.

A combination of methods was used to locate the position in the image of the corners on the specimen for each frame, including a Harris corner detector. Having already fully calibrated the camera for each mirror, these pixel positions were then used to triangulate the 3D position of each of the corners throughout the experiment, as shown in Figure 2.
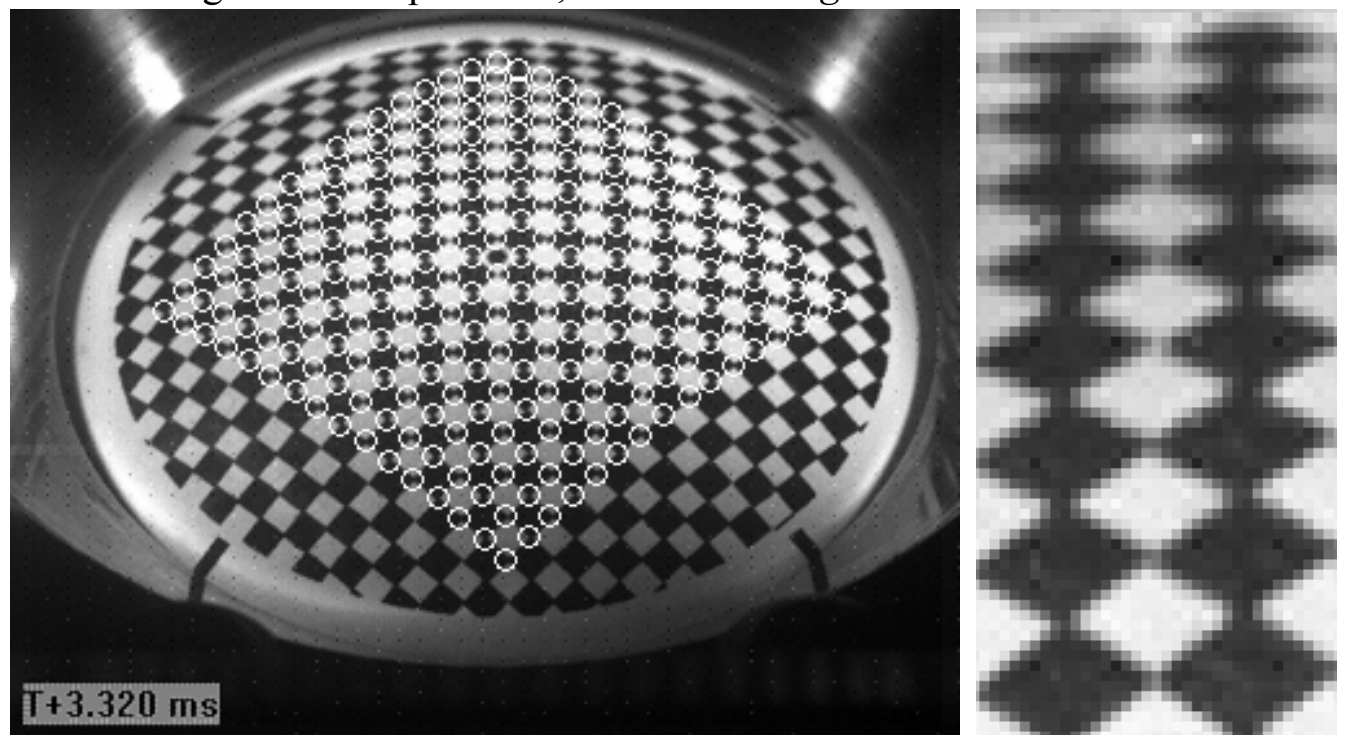

Figure 1: An example of the results of a bulge test with the corners that were used in the analysis highlighted (left) with an area affected by blurring zoomed in (right)
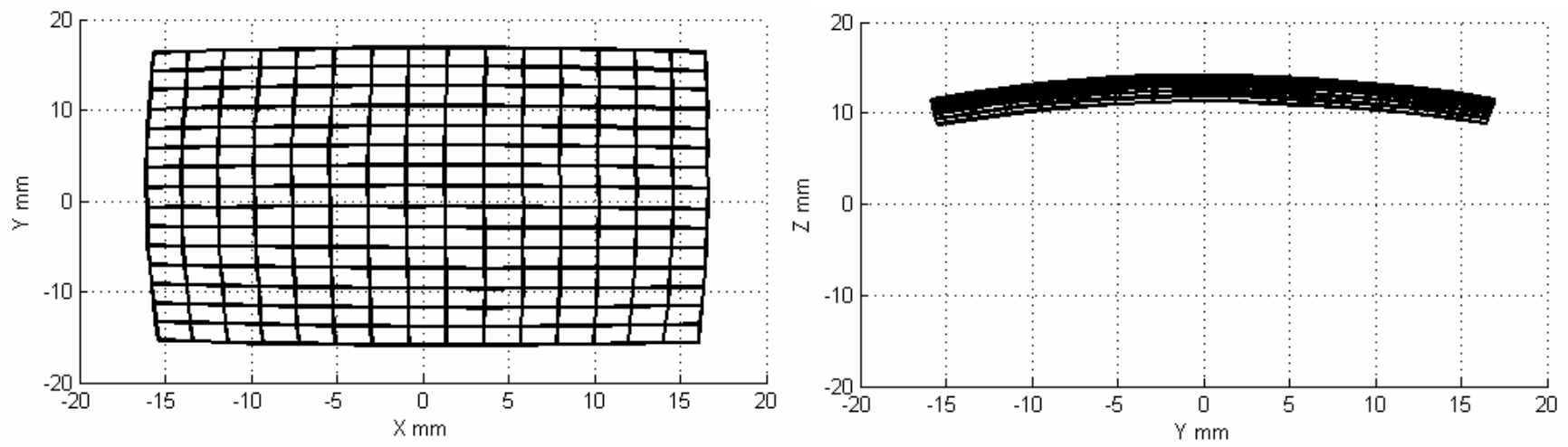

Figure 2: An example of the results of the analysis, showing the triangulated $3 D$ data 
There are various problems with performing and photographing experiments at high-speed. Due to the lighting requirements necessary for the very short shutter speeds that come with high speed filming, a large aperture is used to allow enough light for the sensor. This means that the depth of field may not be large enough to cover the displacement of the specimen throughout the entire experiment. This may cause some blurring to occur as the right image of Figure 1 demonstrates.

\section{Error analysis of corner detection methods}

The work on the bulge tests inspired a more general study and error analysis of targets and target detection within impact engineering. This paper will only include the results on corners when investigating the effect of blurring.

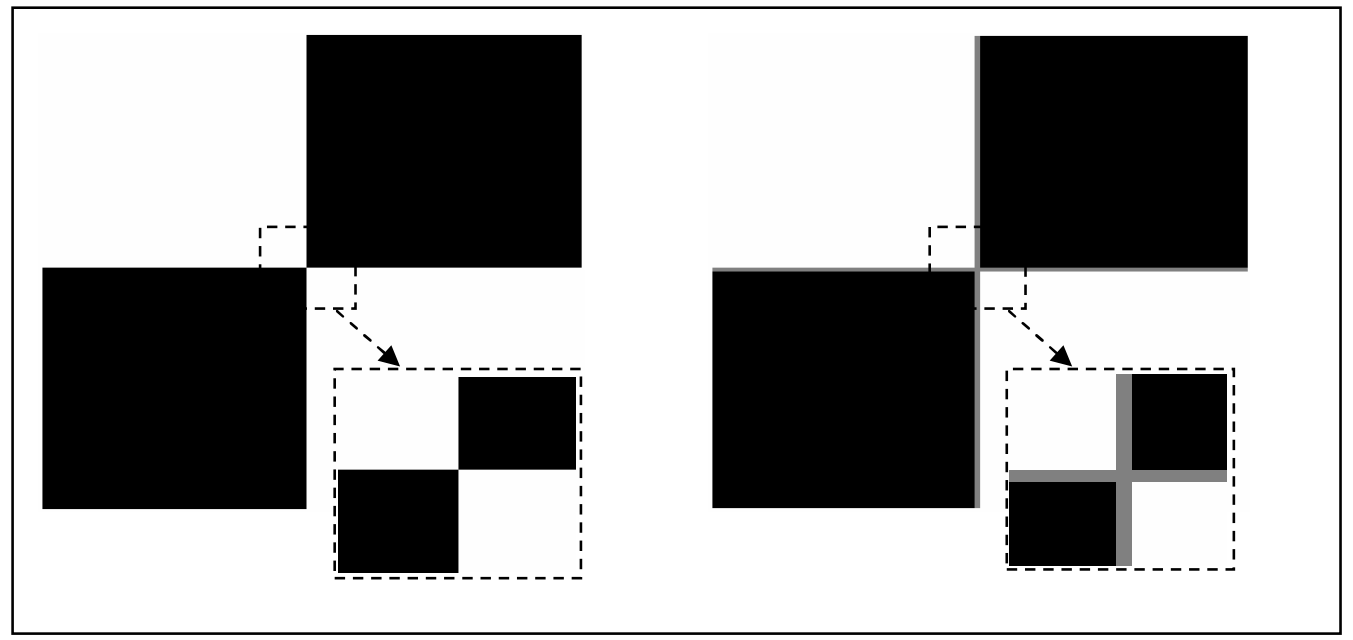

Figure 3: A corner that lies exactly on a pixel (left) and one that lies between pixels (right)

The edges in the corners are considered as step functions, from the colour of the target to the colour of the background. For the purposes of this error analysis, the percentage of area within each pixel that was covered by the corner determines the intensity of that pixel. For example: if a black section of the corner completely covers a pixel, it will be shaded completely black (0); if a white section of the corner completely covers a pixel, it will shaded completely white (1); and if a section of a corner covers $50 \%$ of a pixel, then it will be shaded mid level grey (0.5). This allows subpixel adjustment of the target so that when a corner moves by a fraction of a pixel, the resultant image reflects any subpixel changes by making the appropriate pixels the correct shade of grey, as shown in Figure 3.

\section{Methodology}

All images were 100 by 100 pixels in size. Several distortions to the images were tested, including resolution, shot noise and detector noise, examples of which are shown in Figure 4, but this study will only analyse the effect of blurring.
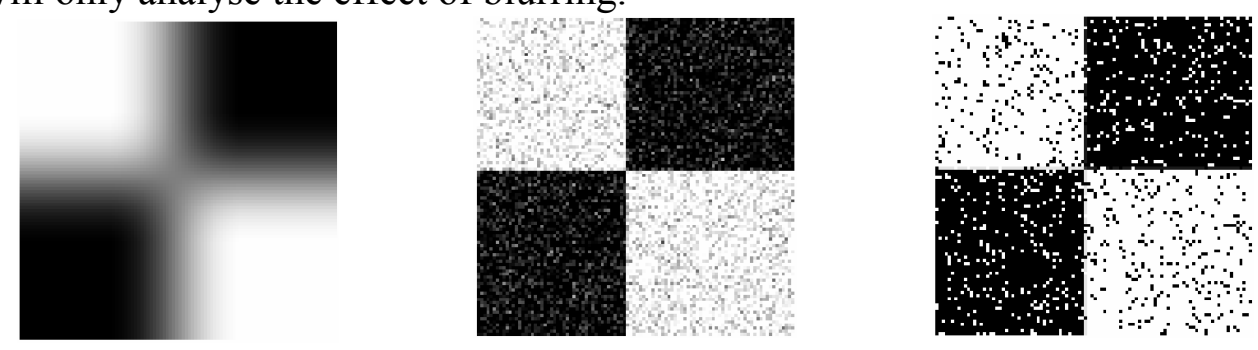

Figure 4: (Left to right) A corner with a large amount of blur; a large amount of detector noise; and a large amount of shot noise (salt and pepper noise) 
Blurring was simulated by convoluting the image with a Gaussian kernel of varying standard deviation, $\sigma$. This simulates one type of error that poor focus (either due to the movement of the object perpendicular to the image plane, insufficient depth of field) may add to the system. The size of the kernel was adjusted by varying its standard deviation, measured in pixels. For this study, the amount of blur is the size of the Gaussian kernel in pixels and the standard deviation is kept a constant third of this value.

Each test consisted of 121 runs with the position of the corner being changed by 0.1 of a pixel between 51.0 and 52.0 in both the $\mathrm{x}$ and $\mathrm{y}$ directions in each run to create a $2 \mathrm{D}$ grid of tests.

The output of each of the methods of locating the corner was compared to the real position of the corner and the mean, maximum and minimum errors were calculated.

The template. The correlation based methods require a template over which to search the target image. These were the same as the image of the corner but with no noise or blur added and created so that the corner always lay on integer pixel positions. Changing the template affects the correlation surface and so the templates were kept constant throughout the analysis while the target image was blurred. The template was a corner located at $(10.0,10.0)$ in an image of size 20 pixels by 20 pixels.

\section{Results}

The correlation surfaces. The left of Figure 5 shows an example of a correlation surface typical of that produced in this study and on the right is a cross section of this correlation surface.
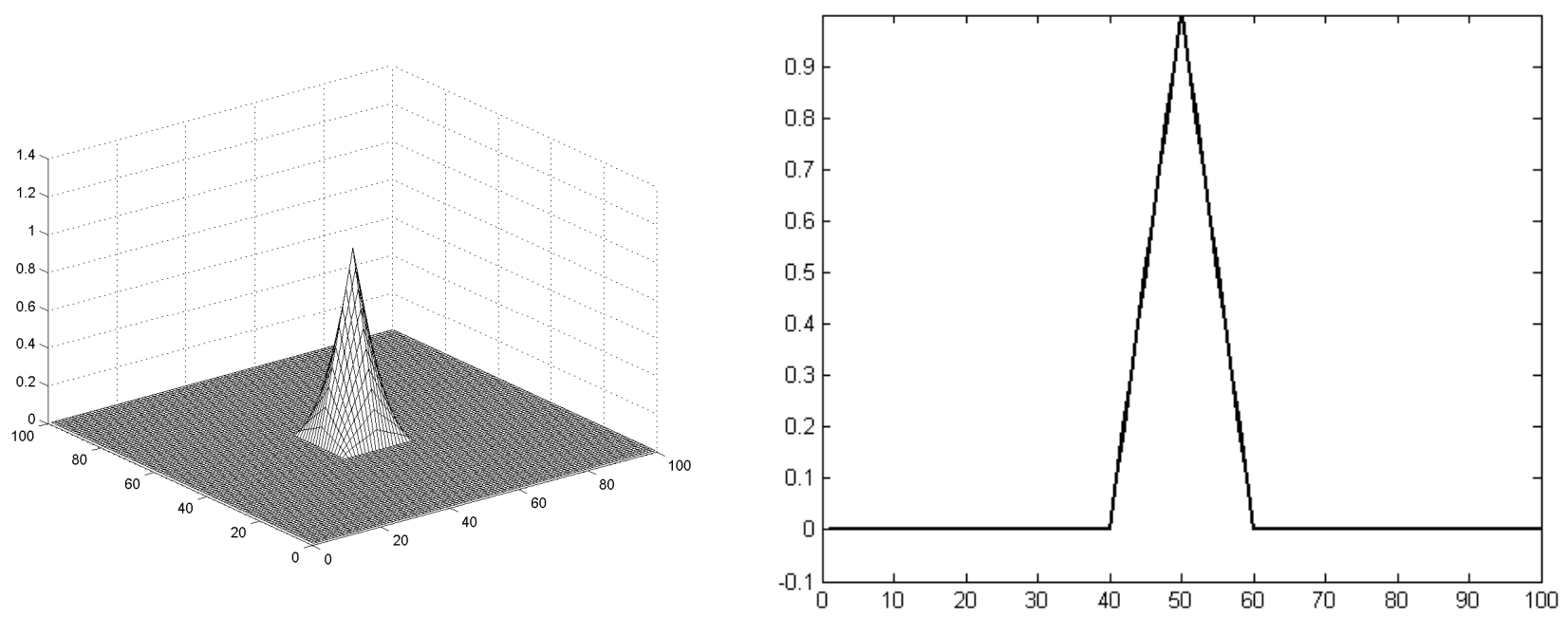

Figure 5: A correlation surface produced by an image of a corner being cross correlated by a template of a corner (left) along with it cross section across the peak (right).

Accuracy when finding the corner to a subpixel resolution. All of the methods of locating a corner reacted in a similar manner to the effect of increasing blur as Figure 6 shows. The error was initially high when there was no blur but it quickly fell when there was small degree of blurring applied, after which it tended to a stable amount of error with increasing blur. The peak of the continuous correlation surface (as shown by the dashed line in Figure 6 ) was unique in having a minimum when a Gaussian kernel with a size of 4 pixels (see Methodology for more information) is convoluted with the image. This is because when there is no blur, the intensities at the edge of the corner have a sharp discontinuity (which can be observed in the cross section of Figure 5), because the edge of each corner is modelled as a step change. This is reflected in the correlation surface when the target image is cross correlated with the template. This sharp discontinuity in the correlation surface causes difficulties when fitting a Fourier series to it, with a ringing effect at the 
sharp discontinuity. With blur, the edges of the target become more spread out and the correlation surface becomes smoother, which means that the Fourier series will have a better fit. The Gaussian fit of the correlation peak has the smallest error.

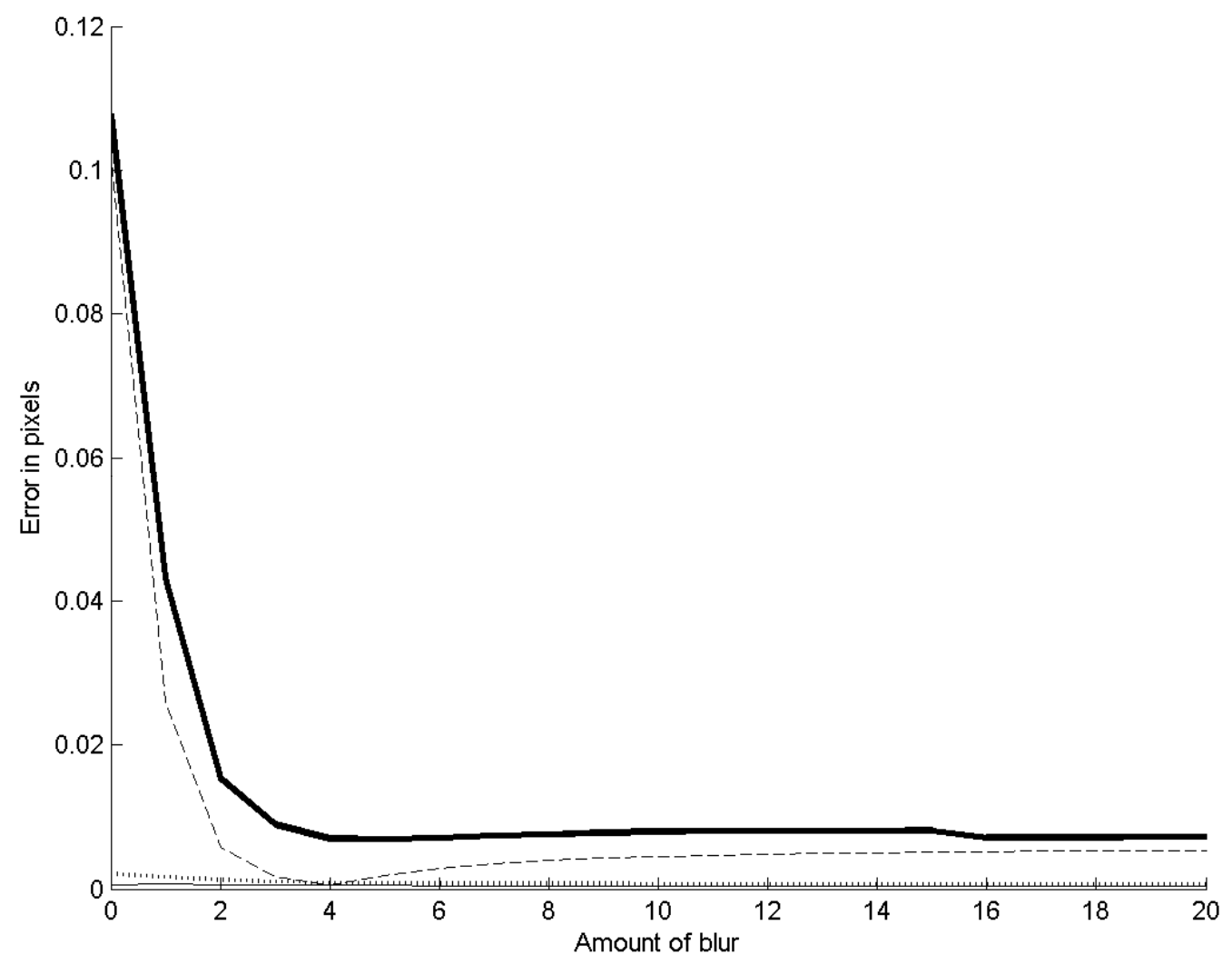

Figure 6: The maximum error when testing the effect of blur: Dashed line-peak of continuous correlation surface; Dotted line - centroid of correlation peak; Solid line - Gaussian fit of correlation peak; Bold solid line - Harris corner detector

Error bias. An attempt was also made at investigating the error bias within a pixel. Presented, is a discussion when the size of the Gaussian kernel used to blur the target image is 9 pixels (see Methodology for more information). The contour maps shows how the error changes as the position of the target is changed with the pixel. The error in pixels is quantified by the colour bar to the right of each contour map.

(a)

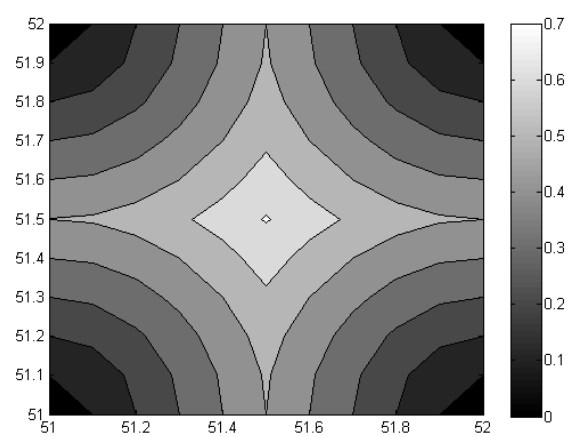

(b)

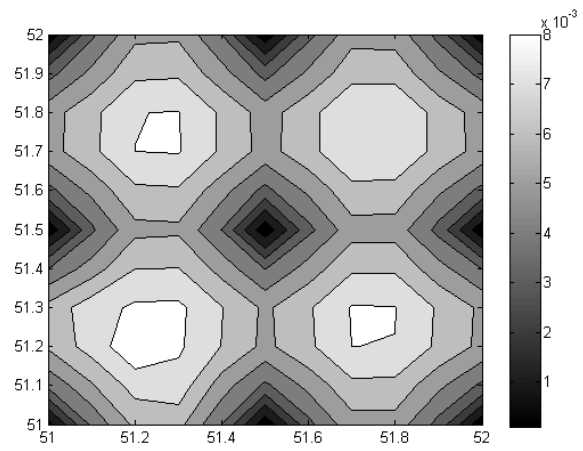

Figure 7: The errors within the pixel when using the discrete correlation peak (a) and the Harris corner detector (b) to locate the corner

The error map on in Figure 7(a) illustrates how the error changes when the peak of the discrete correlation surface is used to locate a corner as the corner changes its position within a pixel. These results are as expected: when the target lies directly on the pixel boundary (at the 
corners of the pixel), there is no error. The error then increases as the target is moved away from the boundaries until it reaches a maximum when the target is positioned at the furthest point from a pixel boundary (when the corner is directly in the centre of a pixel).

Figure 7(b) shows the error when the Harris corner detector is used to locate a corner as it varies position within a pixel. All the subpixel methods produce error maps similar to this. The error map has roughly symmetrical errors within a pixel with the maxima of errors at: $(51.2,51.8),(51.8$, $51.2),(51.2,51.2)$ and $(51.8,51.8)$, i.e. when the corner is at the most anti-symmetrical position within the pixel. Similarly, when the corner is located in positions within the pixel that make the target close to symmetrical, the error is minimised. It is slightly biased towards the bottom left corner of the pixel (whereas the other correlation based methods are not when they are used to locate a corner) because this implementation requires an initial estimate of the position of the target as an input. The implementation then searches for a corner within the neighbourhood of this initial estimate, but biases any possible location by their distance to the initial estimate.

\section{Further applications}

The bulge tests performed on Ti64 was mentioned above as an example of an experiment that motivated this error analysis. Since then numerous other experiments at high rate have been performed that have benefitted from this deeper understanding of the errors present. These include: testing to emulate the effect of birds on fan blades; investigating fast crack initiation in a panel specimen; and even full scale bird impact tests on a single fan blade. Figure 8 shows example frames from some of these experiments.

(a)

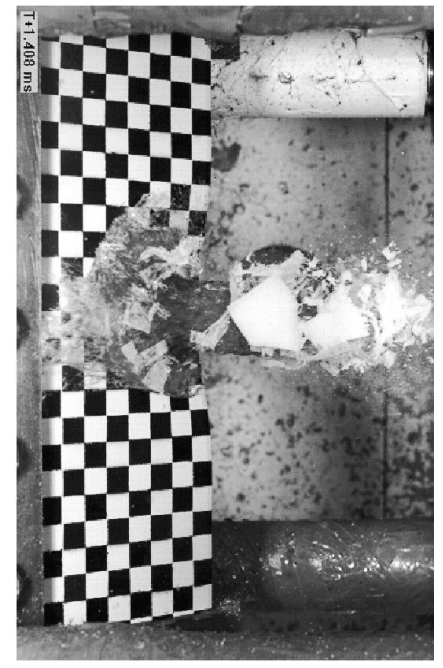

(b)

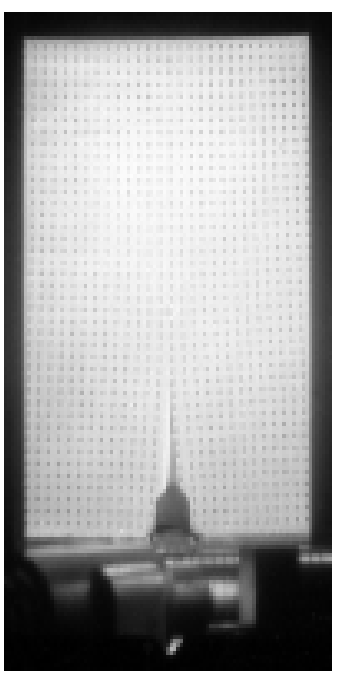

Figure 8: (a) Testing to emulate the effect of birds on fan blades; (b) fast crack initiation in a panel specimen.

\section{Conclusions}

Fitting a Gaussian function to the discrete correlation peak was found to be one of the most accurate methods when varying the amount of blur on a corner target image. This is due to the type of correlation surface that is produced when a larger image corner is cross correlated with a smaller template corner, as shown in Figure 5. This sharp peak lends itself to being modelled by a Gaussian function.

Using a Fourier series expansion of the discrete correlation surface, to create a continuous correlation surface and finding its peak to sub-pixel accuracy, as described in [2], was found to be robust to noise and able to find the location of targets to a high accuracy. When there is no blur, the correlation surfaces produced have sharp discontinuities due to the abrupt changes in intensity in the 
image. This was found to have an effect on the fitting of the Fourier series to the discrete correlation surface and so the amount of error when finding the peak of the continuous correlation surface. This is described in above in Results.

The Harris corner detector is a specialised method that is intended to find targets similar to corners. It was found to be accurate but this implementation had more error than the other methods. It is often used in computer vision in conjunction with other methods or constraints, for example epipolar constraints or as the basis of very sophisticated object recognition systems [5].

\section{References}

1. Fraser, C.S. and B. Riedel, Monitoring the thermal deformation of steel beams via vision metrology. Isprs Journal of Photogrammetry and Remote Sensing, 2000. 55(4): p. 268-276.

2. Sjodahl, M. and L.R. Benckert, Electronic Speckle Photography - Analysis of an Algorithm Giving the Displacement with Subpixel Accuracy. Applied Optics, 1993. 32(13): p. 22782284.

3. Chevalier, L., et al., Digital image correlation used to analyze the multiaxial behavior of rubber-like materials. European Journal of Mechanics a-Solids, 2001. 20(2): p. 169-187.

4. Harris, C. and M. Stephens. A Combined Corner and Edge Detector. in The Fourth Alvey Vision Conference. 1988. Manchester.

5. Sivic, J., F. Schaffalitzky, and A. Zisserman, Object level grouping for video shots. Computer Vision - Eccv 2004, Pt 2, 2004. 3022: p. 85-98.

6. Bouget, J.-Y., Camera Calibration Toolbox for Matlab. 2006. 